\title{
Low Radiation Environment Switches the Overgrowth-Induced Cell Apoptosis Toward Autophagy
}

\section{OPEN ACCESS}

Edited by:

Claudio Pioli,

Italian National Agency for New Technologies, Energy and Sustainable Economic Development (ENEA), Italy

Reviewed by:

Francis A. Cucinotta, University of Nevada, Las Vegas,

United States

Paul Gottlob Layer,

Darmstadt University of

Technology, Germany

*Correspondence:

Alessandra Tessitore

alessandra.tessitore@univaq.it

tThese authors have contributed equally to this work

Specialty section:

This article was submitted to

Radiation and Health

a section of the journal

Frontiers in Public Health

Received: 14 August 2020 Accepted: 26 November 2020 Published: 12 January 2021

Citation:

Fischietti M, Fratini E, Verzella $D$,

Vecchiotti $D$, Capece D, Di

Francesco B, Esposito G, Balata M,

loannuci L, Sykes $P$, Satta $L$,

Zazzeroni F, Tessitore A,

Tabocchini MA and Alesse E (2021)

Low Radiation Environment Switches

the Overgrowth-Induced Cell

Apoptosis Toward Autophagy.

Front. Public Health 8:594789.

doi: 10.3389/fpubh.2020.594789

\begin{abstract}
Mariafausta Fischietti ${ }^{1,2}$, Emiliano Fratini ${ }^{1,3}$, Daniela Verzella ${ }^{2}$, Davide Vecchiotti ${ }^{2}$, Daria Capece' ${ }^{2}$, Barbara Di Francesco ${ }^{2}$, Giuseppe Esposito ${ }^{3,4}$, Marco Balata $^{5}$, Luca loannuci ${ }^{\text {, }}$ Pamela Sykes ${ }^{6}$, Luigi Satta ${ }^{1}$, Francesca Zazzeroni ${ }^{2}$, Alessandra Tessitore ${ }^{2 *}$, Maria Antonella Tabocchini ${ }^{1,34}{ }^{4}$ and Edoardo Alesse ${ }^{2 t}$

${ }^{1}$ Museo Storico della Fisica e Centro Studi e Ricerche Enrico Fermi, Rome, Italy, ${ }^{2}$ Department of Biotechnological and Applied Clinical Sciences, L'Aquila University, L'Aquila, Italy, ${ }^{3}$ Istituto Superiore di Sanità, National Center for Innovative Technologies in Public Health, Rome, Italy, ${ }^{4}$ Istituto Nazionale di Fisica Nucleare (INFN) Sezione Roma, Rome, Italy, ${ }^{5}$ INFN-Gran Sasso National Laboratory, Assergi L'Aquila, Italy, ${ }^{6}$ Flinders Center for Innovation in Cancer, Flinders University, Adelaide, SA, Australia
\end{abstract}

Low radiation doses can affect and modulate cell responses to various stress stimuli, resulting in perturbations leading to resistance or sensitivity to damage. To explore possible mechanisms taking place at an environmental radiation exposure, we set-up twin biological models, one growing in a low radiation environment (LRE) laboratory at the Gran Sasso National Laboratory, and one growing in a reference radiation environment (RRE) laboratory at the Italian National Health Institute (Istituto Superiore di Sanità, ISS). Studies were performed on pKZ1 A11 mouse hybridoma cells, which are derived from the pKZ1 transgenic mouse model used to study the effects of low dose radiation, and focused on the analysis of cellular/molecular end-points, such as proliferation and expression of key proteins involved in stress response, apoptosis, and autophagy. Cells cultured up to 4 weeks in LRE showed no significant differences in proliferation rate compared to cells cultured in RRE. However, caspase-3 activation and PARP1 cleavage were observed in cells entering to an overgrowth state in RRE, indicating a triggering of apoptosis due to growth-stress conditions. Notably, in LRE conditions, cells responded to growth stress by switching toward autophagy. Interestingly, autophagic signaling induced by overgrowth in LRE correlated with activation of p53. Finally, the gamma component of environmental radiation did not significantly influence these biological responses since cells grown in LRE either in incubators with or without an iron shield did not modify their responses. Overall, in vitro data presented here suggest the hypothesis that environmental radiation contributes to the development and maintenance of balance and defense response in organisms.

Keywords: low radiation environment, LRE, apoptosis, autophagy, PARP1, p53

\section{INTRODUCTION}

Life has evolved on Earth for more than three billion years in ecosystems characterized by different levels of environmental radiation. This abiotic factor, acting as a natural tiny but constant daily stimulus, is at the heart of the development of life and has been incorporated within the biology of organisms during evolution (1). However, very little is known about molecular mechanisms 
underpinning the effects of this influencing factor on living beings. To this aim, analysis of differences between two parallel biological systems, one kept in a reference radiation environment (RRE) and one in a very low radiation environment (LRE), helps to enhance knowledge in this field. During the last decades, studies performed in underground laboratories highlighted that biological models could react to background radiation changes in different ways. It was demonstrated that behavior of living systems under low radiation dose exposure can lead to interesting, and often unexpected, results. Several effects, such as genomic instability, with increase of DNA changes through generations; transgenerational effects, with hereditary alterations; bystander effect, which causes damage of healthy cells near to those irradiated, have been observed (2-4).

The peculiar location of the underground Gran Sasso National Laboratory (LNGS) of the Italian Institute of Nuclear Physics (INFN), characterized by very low-radiation conditions, makes it particularly suitable for implementing not only studies in the field of physics, such as proton decay or solar neutrino detection, but also in that of biology. In this location, shielded by more than 1400 meters of carbonate (dolomia) rock, cosmic radiation is almost completely absent (5). Furthermore, due to the nature of the rocks, Uranium and Thorium are barely detected, and the neutron flux is reduced by a factor of $10^{3}$ with respect to external values (6). In addition, radon concentration is kept at a very low level by an efficient ventilation system that pumps air from the outside into the laboratory.

Accordingly, the low LET components, especially photons, are predominant at LRE. On the other hand, at RRE, there are both low LET and high LET components.

Previous researches demonstrated the influence of different environmental radiation on biological systems of different origin (yeast, mouse, and human), indicating that cell cultures maintained in LRE, compared to those in RRE, developed a different biochemical response, being less preserved from DNA damage and showing reduced Reactive Oxygen Species (ROS) scavenging power (1, 7-9).

In this study, we took advantage of the Gran Sasso National Laboratory to further show the core mechanisms putatively responsible for different cellular behaviors attributable to LRE. In particular, we investigated the molecular response of the pKZ1 A11 mouse hybridoma cells $(10,11)$, cultured in parallel in LRE (at LNGS) and in RRE (at ISS) laboratories, to overgrowth stress conditions, in which cells undergo nutrient deprivation, hypoxia, and $\mathrm{pH}$ unbalance. We found that radiation environment does not affect cell proliferation both in exponentially growing cells and in overgrowth conditions. On the contrary, cells overgrowing in LRE were prone to respond to this stress by inducing autophagy instead of apoptosis, which was not observed in cells parallelly cultured in RRE.

\section{MATERIALS AND METHODS}

\section{Cell Lines and Proliferation Curve}

The hybridoma A11 mouse cell line, which contains a pKZ1 chromosomal inversion cassette $(10,11)$ was used for the experiments. Cells were cultured under identical conditions in
RPMI 1640 (Gibco) supplemented with 5\% FBS, L-glutamine, and penicillin-streptomycin at $37^{\circ} \mathrm{C}$ in a $5 \% \mathrm{CO}_{2}$ humidified incubator. For the evaluation of the role of gamma component, cells were further cultured in parallel in two different incubators, with or without an iron shield, which is able to reduce the gamma component of the radiation spectrum by a factor of about four. For each experiment, parallel cell cultures were plated at $2 \times$ $10^{4}$ cells $/ \mathrm{mL}$ in $25 \mathrm{~mL}$ of complete medium in $75 \mathrm{~cm}^{2}$ flasks and grown in quadruplicate for up to 4 weeks at the LNGS Gran Sasso underground laboratory and at the ISS, under low (LRE) and reference (RRE) environmental radiation, respectively. Cells were passaged every Monday ( $72 \mathrm{~h}$ of culture) and every Friday ( $96 \mathrm{~h}$ of culture). An aliquot of $0.5 \mathrm{~mL}$ of cells was diluted 1:10 with Isoton Diluent (Beckman Coulter) and counted using an automatic cell counter (Coulter Counter). At every cell passage (every $72 \mathrm{~h}$ and $96 \mathrm{~h}$ ), cells were seeded at $2 \times 10^{4}$ cells/ mL in $25 \mathrm{~mL}$ of complete medium in $75 \mathrm{~cm}^{2}$ flasks. Aliquots of the cell suspension were also used to construct a growth curve along a period of 11 days.

After 4 weeks of culture in LRE, cells were moved to the RRE laboratory and grown for 2 more weeks in RRE.

\section{Protein Extraction and Western Blot}

Cells were centrifuged at 1,200 RPM, and protein extracts were prepared at $+4^{\circ} \mathrm{C}$ in RIPA buffer (1x phosphate buffer, $1 \%$ NP40, $0.5 \%$ sodium deoxycholate, $0.1 \%$ SDS, $10 \mu \mathrm{L} / \mathrm{mL}$ PMSF, $30 \mu \mathrm{L} / \mathrm{ml}$ aprotinin; $10 \mu \mathrm{L} / \mathrm{mL}$ sodium orthovanadate) containing complete mini EDTA-free protease inhibitors (Roche Molecular Biochemicals, Mannheim, Germany). Protein concentration was determined by the BCA assay (Pierce, Thermo Fisher). Forty micrograms of proteins were loaded onto a SDS-PAGE and subjected to electrophoresis, and then proteins were electro-transferred to a nitrocellulose membrane (Whatmann, Dassel, Germany) and hybridized to anti-PARP1 (1:1,000; Cell Signaling Technology, cat \#9542), anti-p53 (1:1,000; Cell Signaling Technology, cat \#2524), anti-P-Ser392-p53 (1:1,000; Cell Signaling Technology, cat \#9281), anti-P-Ser15-p53 (1:1,000, Cell Signaling Technology, cat \#9284), anti-HSP70 (1:1,000 Cell Signaling Technology, cat \#4876), anti-Caspase 3 (1:1,000, Cell Signaling Technology, cat \#9662), anti-LC3B (1:1,000, Thermofisher, cat. \#PA5-32254, which recognizes both LC3BI and II), anti-actin (1:2,000 Santa Cruz Biotech, cat sc-8432). Membranes were then incubated with specific horseradish peroxidase-conjugated secondary antibodies (Santa Cruz Biotech). Protein bands were visualized using a chemiluminescent detection system (Thermo Scientific, Rockford, USA).

\section{Densitometric Analysis and Statistics}

Densitometric analysis of immunoblotting was performed by using the ImageJ software (Rasband, W.S., ImageJ, U.S. National Institutes of Health, Bethesda, Maryland, USA, https://imagej. nih.gov/ij/, 1997-2018). All densitometries were expressed as a ratio of analyzed protein to endogenous control expression. Due to the demanding and very peculiar underground working conditions, it was possible to iterate just some immunoblotting experiments (i.e., anti-P-p53 Ser392, anti-PARP-1, anti-Caspase 3) for which statistical elaboration was provided. Results were 
expressed as mean of two independent experiments \pm SD. Data were analyzed using the GraphPad Prism (7.0 version) software and statistical analysis of the results was performed using the 2 -tailed Student's $t$-test. $P<0.05$ were considered statistically significant.

\section{RESULTS}

\section{Setting Up of Experimental Conditions}

In order to evaluate the effect of LRE on cell behaviors, such as cell proliferation and stress-induced cell death, a pKZ1 A11 hybridoma cell line was chosen as a model. This cell line was obtained by fusion of splenic lymphocytes of pKZ1 mouse with murine multiple myeloma cells P3653 (10). The pKZ1 mouse model has been extensively used to study the effects of low dose radiation on the adaptive response and to identify hormetic mutation responses (12-20). In order to set up the experimental conditions, pKZ1 A11 hybridoma cells were cultured in the RRE laboratory in Rome at the ISS (Figure 1A). Cell proliferation curve displayed a linear growth between 24 to $72 \mathrm{~h}$, showing an inflection at $96 \mathrm{~h}$ and reaching a plateau at $123 \mathrm{~h}$ (Figure 1A). Interestingly, after $96 \mathrm{~h}$, we observed a strong cleavage of PARP1, a known marker of apoptosis (Figures 1B,C) (21-23). Increase of PARP1 cleavage was detected in a time-dependent manner, reaching high level after $96 \mathrm{~h}$, when cells initiated to enter to the proliferation plateau and began to stay in overgrowth stress conditions, due to nutrient and growth factor deprivation, $\mathrm{pH}$ changes of medium, higher oxygen need and consumption. These data indicated that, in RRE, pKZ1 A11 cells are prone to initiate and progress through apoptosis in response to the onset of overgrowth stress conditions.

\section{Growth of pKZ1 A11 Cells Is Not Impaired in Low Radiation Environment}

In order to assess the existence of differential behaviors potentially due to the environmental radiation levels, a twin culture of pKZ1 A11 cells was maintained in parallel in LRE and RRE, exactly under the same conditions, for 4 weeks and split twice a week, every 72 and $96 \mathrm{~h}$. As shown in Figure 2A, no differences were observed in terms of proliferation rate in such conditions between cells grown in RRE and LRE. In accordance with data shown in Figures 1A,B, cell count at $72 \mathrm{~h}$ of each week of culture displayed a number of cells consistent with a linear growth rate, and cell count at $96 \mathrm{~h}$ of each week of culture showed a number of cells consistent with an initial overgrowth plateau state (Figure 2A). Moreover, to evaluate the effect of RRE on cells cultured for 1 month in LRE, pKZ1 A11 cells grown for 4 weeks in LRE were moved to the RRE laboratory and cultured for two more weeks (Figure 2A, weeks 5 and 6). No differences in proliferation were observed. These data showed that LRE does not influence the cell growth behavior of pKZ1 A11 cells.

\section{PARP1 Cleavage Is Strongly Reduced in LRE-Overgrown Cells}

To evaluate the effect of LRE at the molecular levels, cells grown as described in Figure 2A were collected twice a week (after 72 and $96 \mathrm{~h}$ of culture) for 6 weeks and analyzed for PARP1 cleavage. As expected, levels of PARP1 cleavage increased in a time-dependent manner, consistent with that described above (Figure 1B). At the same way, similar behavior was detected in terms of PARP1 expression and cleavage in RRE and LRE under conditions of linear growth $(72 \mathrm{~h})$. Interestingly, lower levels of PARP1 cleaved form were detected in LRE overgrown cells (96h) compared to those cultured in RRE (Figures 2B,C). PARP1 cleaved protein was reduced approximately by half in LRE compared to RRE (Figure 2C). Interestingly, this effect globally reverted when, after 4 weeks of underground culture, cells were moved to the RRE laboratories for 2 more weeks (Figures 2B,C, weeks 5 and 6), indicating a plasticity of cells toward their response to low environmental radiation.

\section{Low Radiation Environment Switches pKZ1 A11 Mouse Hybridoma Response to Overgrowth From Apoptosis Toward Autophagy}

To further shed light on the molecular response of overgrown cells cultured in LRE vs. RRE, we performed the western blot analysis of proteins known to be implicated in cell stress response, such as Heat Shock Protein (HSP) 70; genomic stability control and cell death regulation, such as p53; markers of apoptosis, such as caspase 3; and markers of autophagy, such as LC3B. As shown in Figure 3, HSP70 levels did not differ between LRE- and RRE-cultured cells upon stress stimulation due to overgrowth ( $96 \mathrm{~h}$ of culture). As expected, the total p53 levels were not significantly modulated, whereas the active form of p53, evaluated as phosphorylation in both Ser15 and Ser392, was strongly induced in LRE overgrowth conditions. A canonical function of p53 is the induction of apoptotic response when cells cannot efficiently repair acquired DNA damage $(24,25)$. In addition to this function, p53 has been shown to be a modulator of the autophagic process (26), which is known to promote survival upon starvation stress and maintain metabolic homeostasis through degradation and recycling of intracellular components. Literature showed that autophagy suppresses p53 and, also, p53 activates autophagy as part of its protective function (26). In our experimental settings, expression of active p53 correlated with the conversion of LC3B-I to the lower migrating form LC3B-II which is used as an indicator of autophagy especially in condition of nutrient starvation $(27,28)$, showing that, in LRE conditions, the cell response to overgrowth stress is to switch toward autophagy (Figure 3). Finally, in accordance with PARP1 cleavage data (Figures 2B, 3), apoptotic cell induction in RRE overgrowing cells was confirmed by caspase 3 induction. These data suggest an alternative mechanism of response and defense toward stress/damage induced by longterm cell culture in strongly reduced radiation background.

\section{Gamma Component of Radiation Does Not Affect LRE Overgrowth Cell Response}

To analyze the putative contribution of gamma component in inducing the above-described effects, cells were further grown in parallel in the LRE laboratory in two incubators, with or without iron shield. The iron $(\mathrm{Fe})$ shield can reduce the gamma 
A

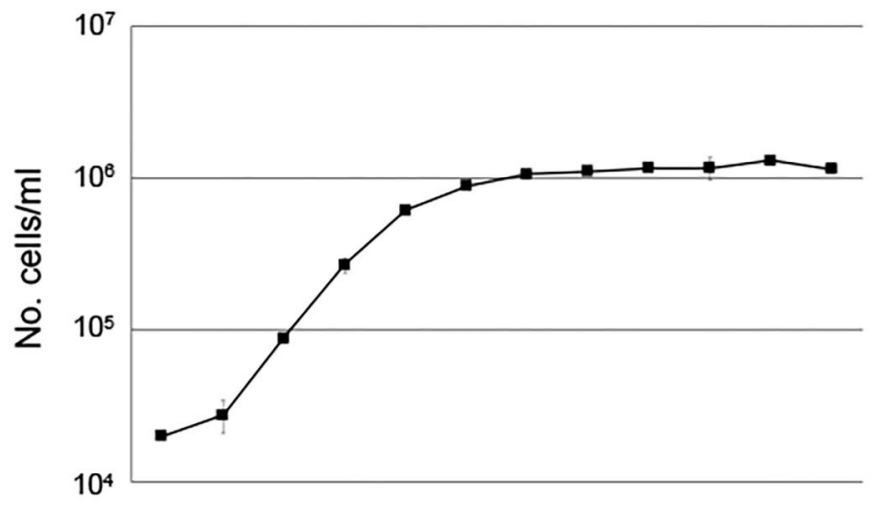

Time (hrs): $0 \quad 24 \quad 48 \quad 72 \quad 96 \quad 123149170191215244264$

B
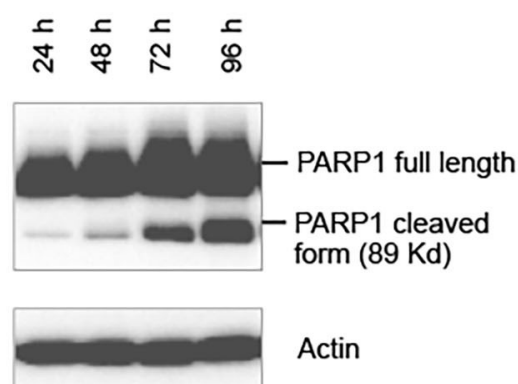

C

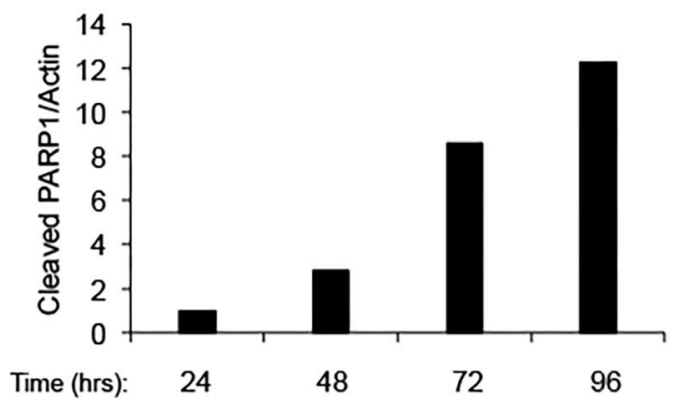

FIGURE 1 | Setting up of pKZ1 A11 mouse hybridoma cells growth condition. (A) Proliferation curve of pKZ1 A11 mouse hybridoma cells cultured at the ISS reference laboratory in Rome. Cells were counted in triplicate. Values are means \pm SD. (B) pKZ1 A11 mouse hybridoma cells cultured as in (A) and analyzed for PARP1 cleavage by western blotting. (C) Densitometry analysis of western blot shown in (B). 
A

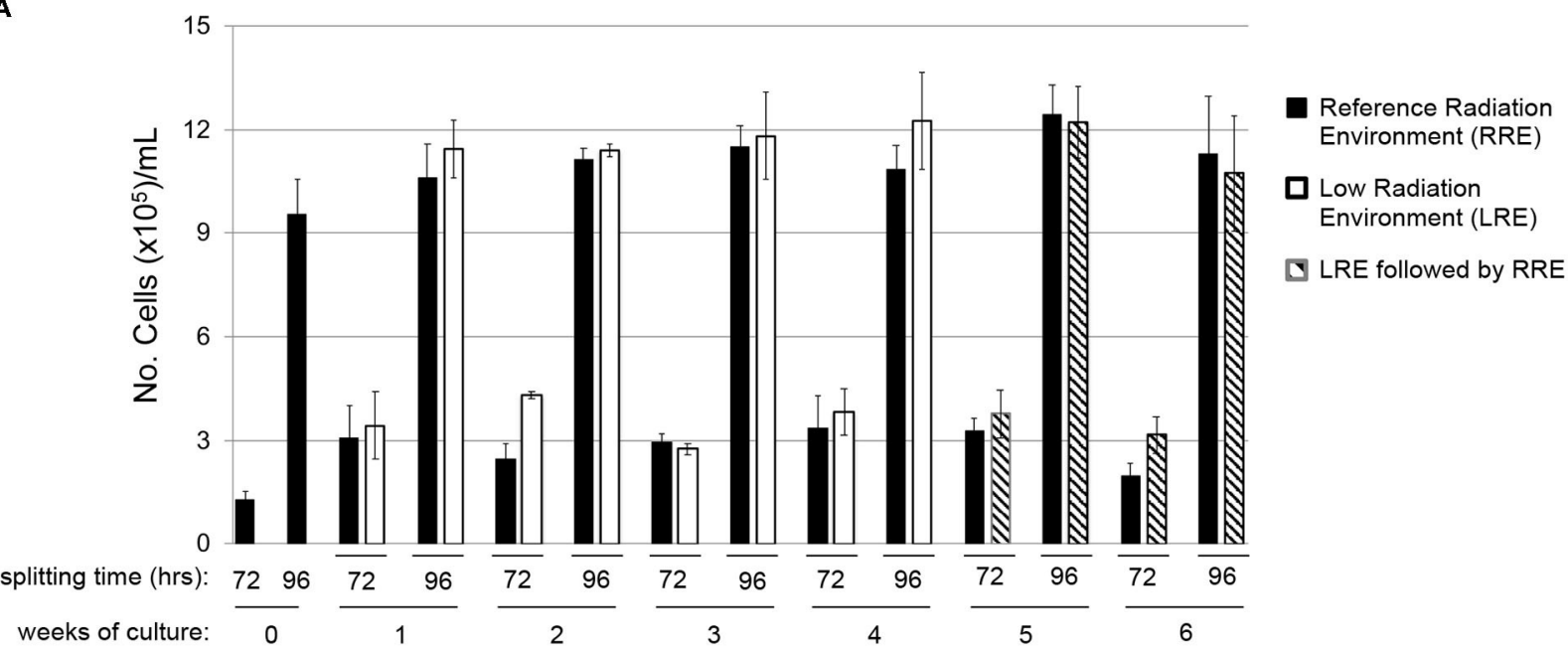

B

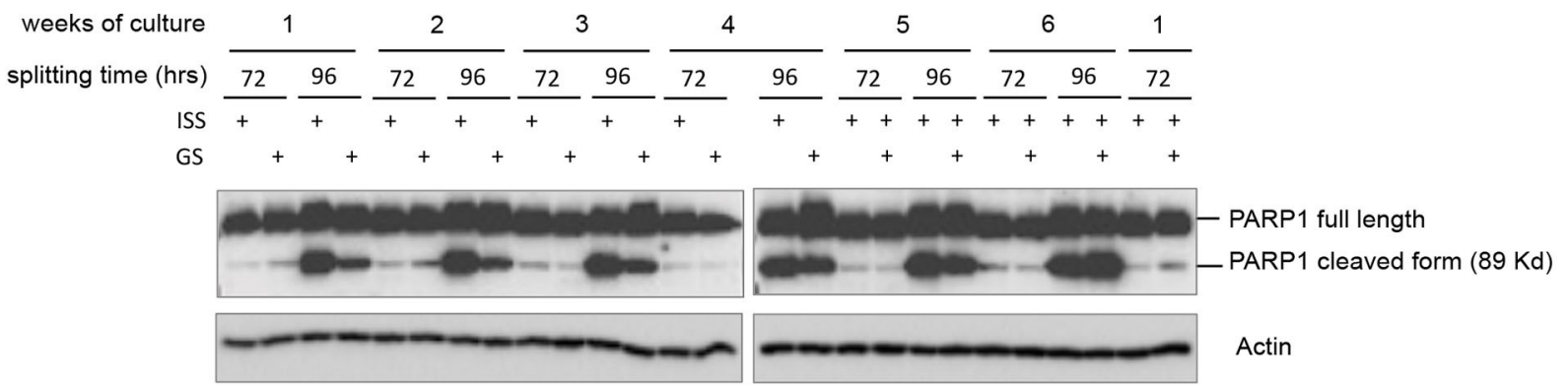

C

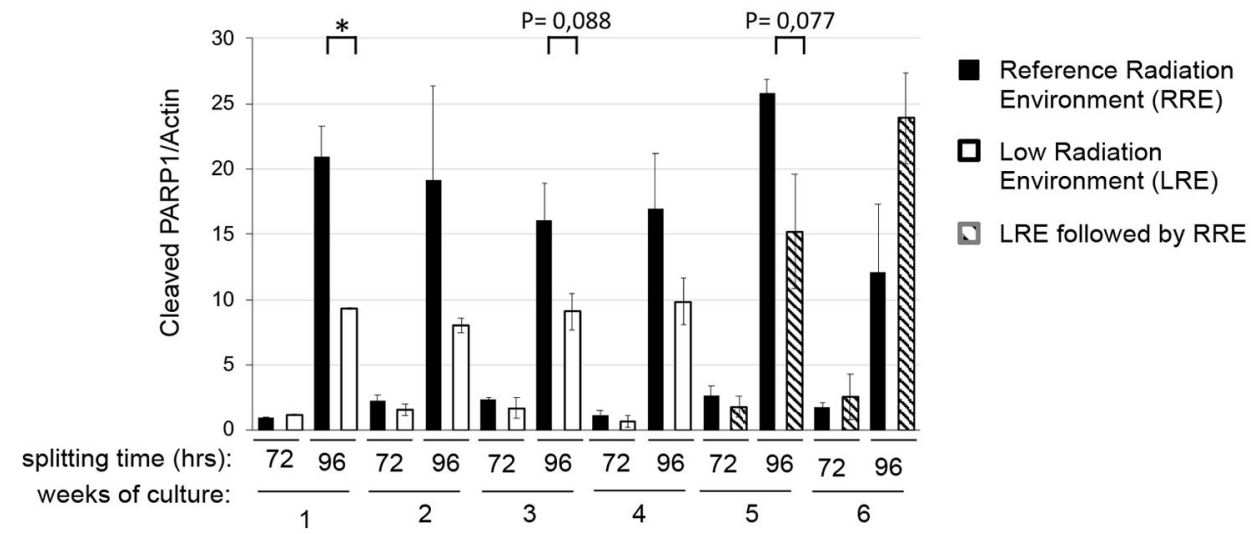

FIGURE 2 | Effects of low radiation environment on pKZ1 A11 mouse hybridoma cell proliferation and overgrowth-induced apoptosis. (A) A twin culture of pKZ1 A11 mouse hybridoma cells was set up in quadruplicate at the ISS reference laboratory in Rome and at the Gran Sasso underground laboratory. Cells were counted and 
FIGURE 2 | split twice a week (after $72 \mathrm{~h}$ and after $96 \mathrm{~h}$ ) for 4 weeks. After 4 weeks of underground cell growth, cells were moved to the ISS-Rome reference lab and grown for additional 2 weeks (weeks 5 and 6). Each cell culture was counted in triplicate. Values are means \pm SD. (B) Western blots showing PARP1 cleavage in pKZ1 A11 mouse hybridoma cells grown as in (A). ISS, Istituto Superiore di Sanità, RRE; GS, Gran Sasso National Laboratory, LRE. (C) Densitometric analysis of anti-PARP1 western blots. Values are means of two independent experiments \pm SD. ${ }^{*} P<0.05$.

component of the radiation spectrum by a factor of about four. As shown in Figure 4A, proliferation of pKZ1 A11 cells was very similar in both conditions. In addition, in presence or absence of iron shielding, a globally comparable protein expression of phospho-p53, PARP1 cleavage and caspase 3 activation, and LC3-II induction was observed (Figures 4B,C), suggesting a negligible role of the gamma component in such cell responses and behavior.

\section{DISCUSSION}

In the last two decades, several efforts have been made by researchers to clarify the roles of low environmental radiations on cell response to stress stimuli. The linear no-threshold (LNT) model was first used in the radioprotection field to predict cell and DNA damage induced by ionizing radiation (Ann. ICRP, 2007). Several studies have, indeed, shown that risk assessment for low radiation dose does not follow a linear curve (29). Interestingly, Non-Target Effects (NTEs) are particularly evident at low doses and include the role of cell communication and responses at the tissue and systemic levels. Among the NTEs, it is possible to highlight the genomic instability and the Bystander Effect (BE), which seem to have a role in increasing the risk of developing cancer above the estimates made by extrapolation of the LNT model, and the Adaptive Response (AR), which, on the contrary, would be a protection against the development of cancer (30). In any case, a common feature of these phenomena is, however, the lack of dose linearity (31). Therefore, shedding light on the molecular mechanisms underlying cell response to low doses of radiation is particularly relevant in the radioprotection field.

In this work, we showed that cells grown in a LRE at the underground Gran Sasso National Laboratory (LNGS) of the Italian Institute of Nuclear Physics (INFN) display a qualitatively different response to stress induced by overgrowth, condition characterized by nutrient and growth factor deprivation, $\mathrm{pH}$ changes of medium, higher oxygen need and consumption. We did not find any difference in the growth of cells cultured in LRE compared to cells cultured in the RRE laboratory. On the other hand, after $96 \mathrm{~h}$ of growth in RRE, we noted an increase of PARP1 cleavage. This was described as an early marker of apoptosis (21). Interestingly, we observed a switch from apoptosis toward autophagy in LRE cultured cells, which appears to be mediated by $\mathrm{p} 53$.

Autophagy is an evolutionarily conserved catabolic pathway involved in many physiological and pathological mechanisms. It induces lysosomal degradation and recycling of non-functional cytoplasm components or organelles, generating substrates which promote stress adaptation and survival. However, autophagy is not intended as just a protective mechanism, as it can lead to an autophagy-associated and autophagy-mediated death, where autophagy occurs by accompanying or inducing apoptosis, or to an autophagy-dependent cell death, which is independent of both apoptosis and necrosis processes (32-34). Nevertheless, molecular mechanisms at the base of connections between autophagy, cell death, and cell fate decision are still unrevealed. Autophagy is increased by cell stress (e.g., DNA damage, hypoxia, starvation, or growth factor deprivation) and depends on complicated signaling networks which include mediators of relevant cell responses, such as $\mathrm{p} 53$, NF- $\kappa \mathrm{B}$, and STAT3 (35). It has been described that, in cancer, autophagy provides a sort of self-eating process to recruit nutrients in a condition where they become unavailable (36). Several studies indicate that stress can induce autophagy through two ways: an early response which takes place within minutes to hours and is essentially mediated by protein post-translational modifications, and a second, delayed response, which is based on specific activation of transcription (37). Moreover, an interesting relationship between p53 and autophagy has been reported, based on the use of in vitro and in vivo preclinical models (26): whereby autophagy can suppress p53 and p53 can activate autophagy. The tumor suppressor p53 usually acts as a tetrameric nuclear transcription factor, inducing genes coding for proteins involved in cell cycle arrest, apoptosis, or autophagy. On the other hand, it was described that cytoplasmic p53 can also promote cell death at the mitochondrial level and suppress autophagy (38). Furthermore, under stress conditions, p53 undergoes post-translation modifications able to avoid $\mathrm{mdm}-2$ driven proteasomal degradation or, alternatively, $\mathrm{mdm}-2$ can be degraded or sequestered by ARF, thus inhibiting the autophagic process. Ser392 is one of the highly conserved phospho-acceptor sites involved in p53 tumor-suppressor activities by enhancing p53 tetramers and DNA binding stabilization (39). Ser392 phosphorylation occurs not only after UV damage, but also after a range of different stimuli, such as etoposide, mdm-2 inhibitors or ionizing radiations (40). Since p53 is a well-known regulator of the apoptotic process too, further experiments will be needed to clarify, in particular, the specific signaling pathway and mechanisms through which p53 induces autophagy instead of apoptosis in our experimental settings.

Data here reported highlight different responses of cells reaching overgrowth stress conditions in RRE or LRE, respectively leading to apoptosis or autophagy, and characterized by the induction of specific mediators/markers involved in those pathways. Those novel results open to further investigations to examine in depth the molecular functional mechanisms specifically activated in RRE or LRE. 
A

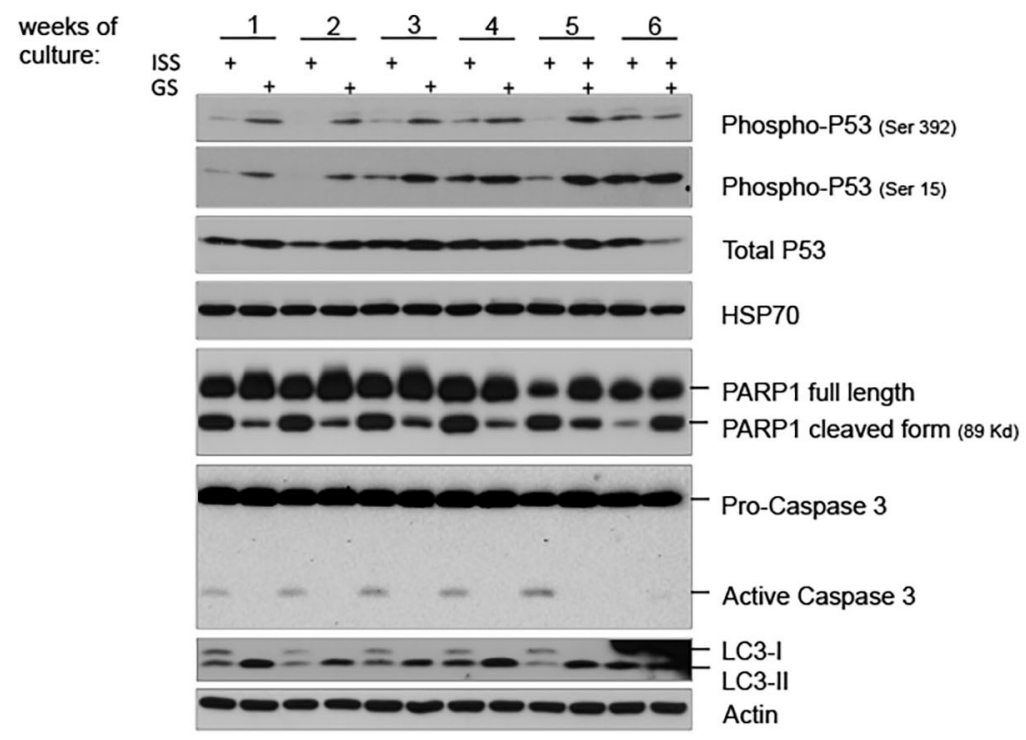

B
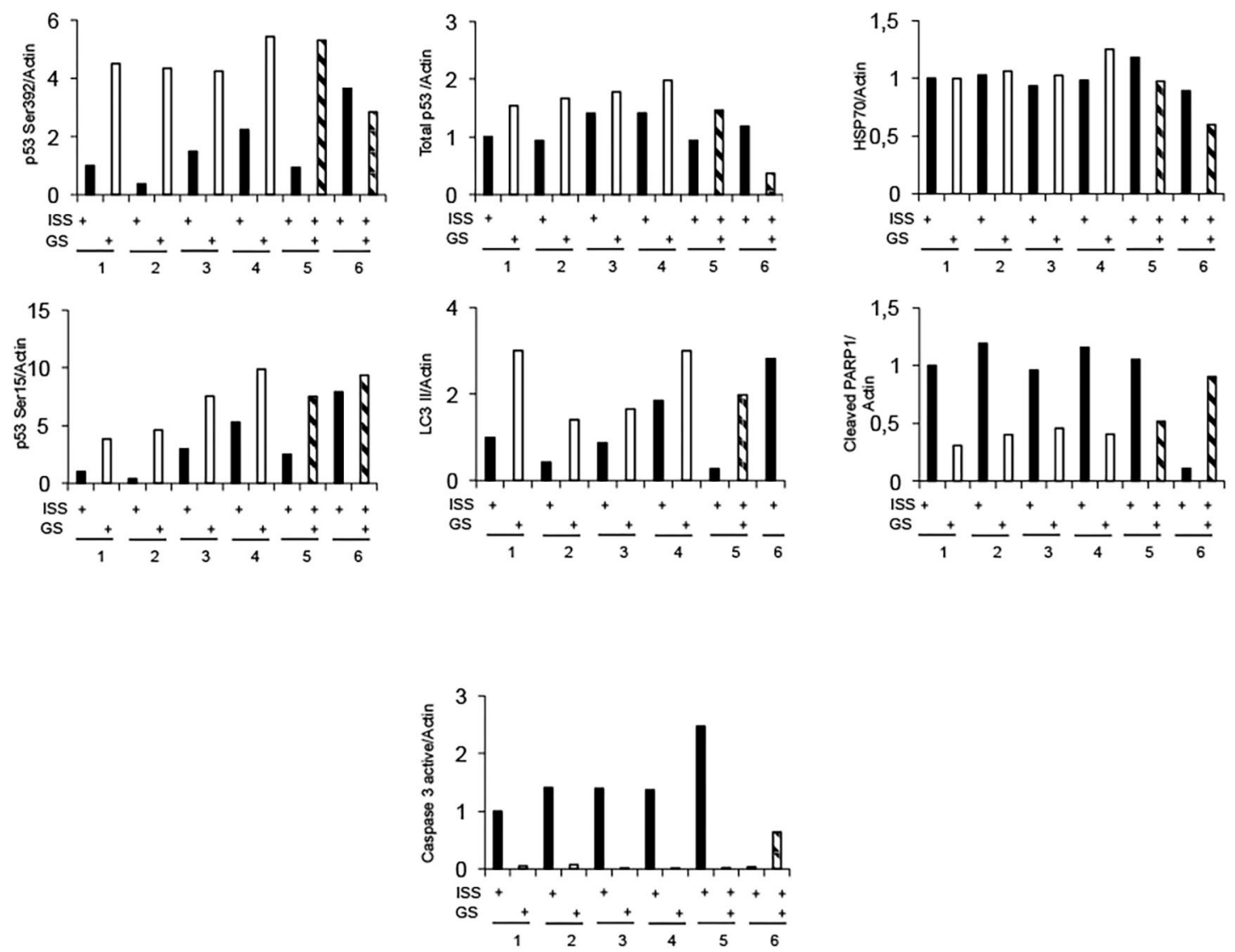

FIGURE 3 | Low radiation environment switches pKZ1 A11 mouse hybridoma overgrowth cell response from apoptosis toward autophagy. (A) Western blots showing activation of p53 and induction of LC3B-II in LRE (LNGS)-grown pKZ1 A11 mouse hybridoma cells and activation of PARP1 and caspase 3 in RRE (ISS) grown pKZ1 A11 mouse hybridoma cells. (B) Densitometry analysis of western blots shown in (A). 
A

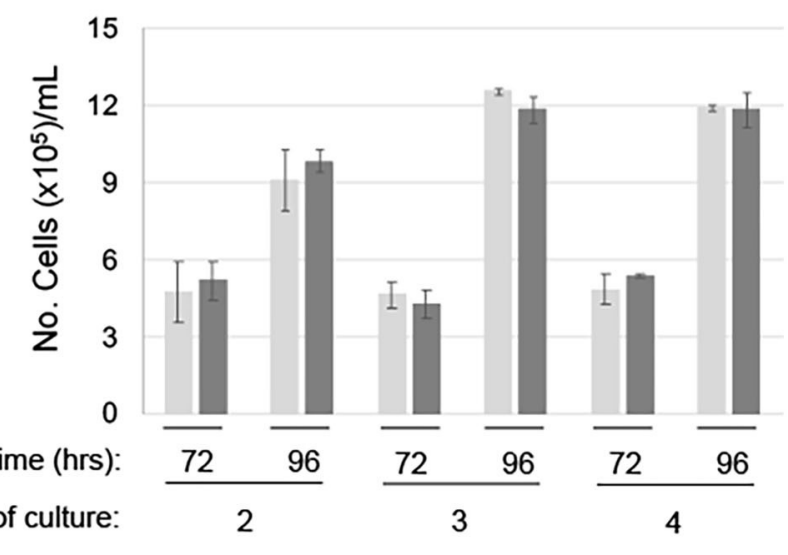

\section{Conventional \\ Incubator $(\mathrm{Cl})$}

Shielded

Incubator (SI)

splitting time (hrs):

weeks of culture:

2

B

\section{C}
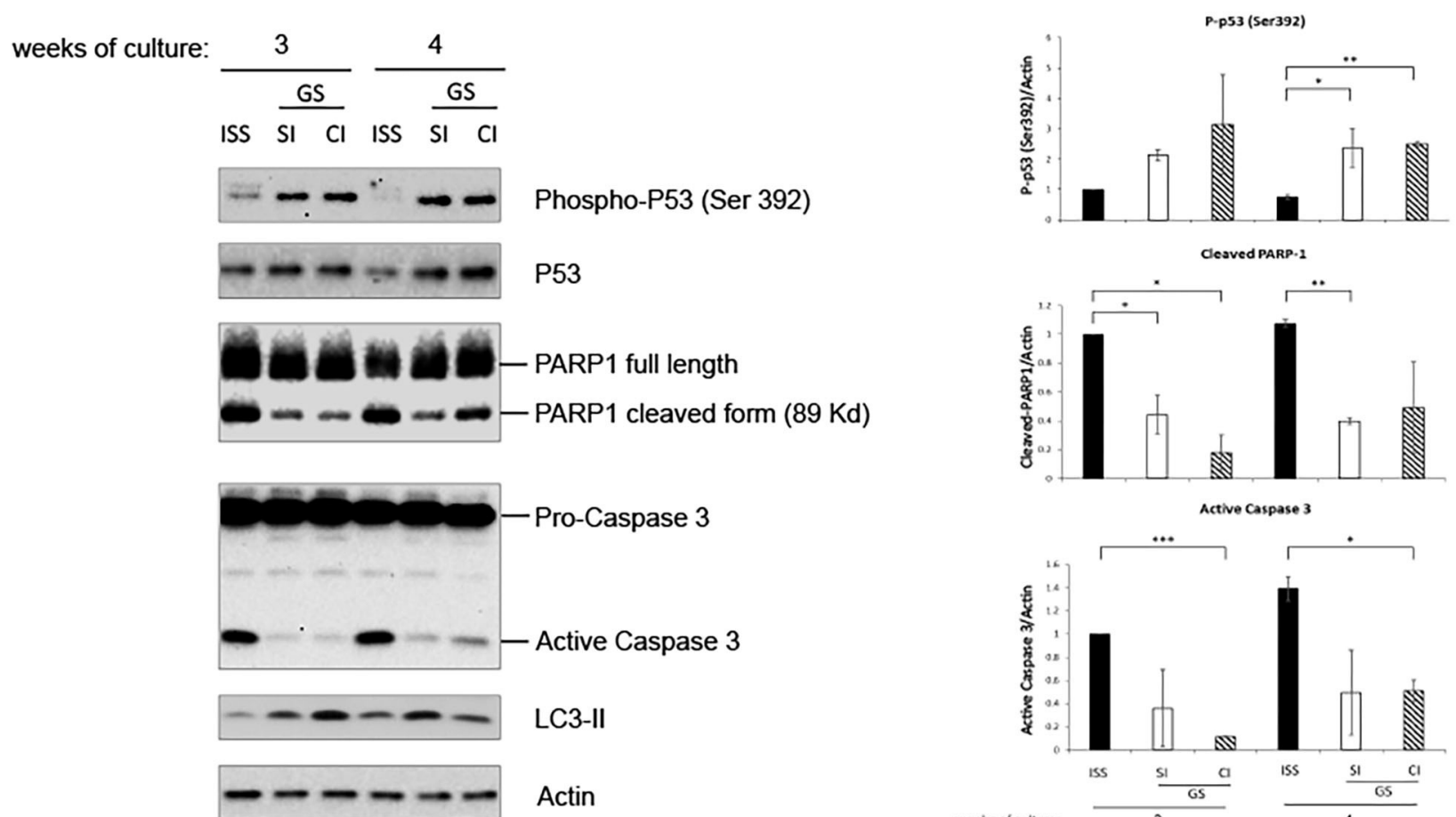

Active Caspase 3

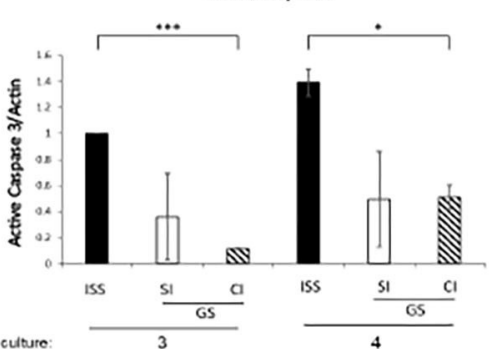

FIGURE 4 | Gamma radiation does not influence pKZ1 A11 mouse hybridoma cell response to overgrowth in low radiation environment. (A) Three cultures of pKZ1 A11 mouse hybridoma cells were set-up in quadruplicate and grown at the ISS reference laboratory in Rome (ISS) and at the Gran Sasso underground laboratory (GS) in a conventional incubator (CI) and in a $\gamma$-radiation shielded incubator (SI). Cells were counted and split twice a week (after $72 \mathrm{~h}$ and after $96 \mathrm{~h}$ ) for 4 weeks. Cells were counted in triplicate. Values are means \pm SD. (B) Cells grown as in (A) were collected after 3 and 4 weeks of cultures (at $96 \mathrm{~h}$ splitting time) and analyzed for apoptotic and autophagic markers by western blots. (C) Densitometric analysis of anti-P-p53 (Ser392), anti-PARP1, anti-caspase3 western blots. Values are means of two different experiments $\pm \mathrm{SD}$. ${ }^{\star} P<0.05,{ }^{* \star} P<0.005,{ }^{\star \star *} P<0.0005$. 
In particular, our findings draw attention to a p53-mediated autophagic cell response in a condition of almost absence of environmental radiation (Gran Sasso National Laboratory) instead of the apoptotic response observed in normal radiation environment. This is particularly fascinating and leaves to hypothesize that environmental radiation levels at the Earth's surface might represent a constitutive low-level stress able to sensitize cells to a prompt response in case of different acute stresses, which could produce further propagation of DNA damage in the case of inefficiently induced apoptosis.

Of further interest is to understand the role of both low and high LET components, both present in the external environment, in determining the response of the biological system. To answer this question, experiments are ongoing at the LNGS, presently focused on the role of the gamma component in the biological response(s) of fruit flies.

\section{DATA AVAILABILITY STATEMENT}

The raw data supporting the conclusions of this article will be made available by the authors, without undue reservation.

\section{REFERENCES}

1. Fratini E, Carbone C, Capece D, Esposito G, Simone G, Tabocchini $\mathrm{MA}$, et al. Low-radiation environment affects the development of protection mechanisms in V79 cells. Radiat Environ Biophys. (2015) 54:183-94. doi: 10.1007/s00411-015-0587-4

2. Mothersill C, Seymour C. Radiation-induced bystander effects: evidence for an adaptive response to low dose exposures? Dose-Response. (2006) 4:28390. doi: 10.2203/dose-response.06-111.Mothersill

3. Morgan WF. Radiation induced genomic instability. Health Phys. (2011) 100:280-1. doi: 10.1097/HP.0b013e3182082f12

4. Mothersill C, Rusin A, Seymour C. Low doses and non-targeted effects in environmental radiation protection; where are we now and where should we go? Environ Res. (2017) 159 484-90. doi: 10.1016/j.envres.2017. 08.029

5. MACRO Collaboration, Ahlen SP, Ambrosio M, Auriemma G, Baldini A, Barbarino GC, et al. Study of penetrating cosmic ray muons and search for large scale anisotropies at the Gran Sasso Laboratory. Phys Lett B. (1990) 249:149-56. doi: 10.1016/0370-2693(90)90541-D

6. Rindi A, Celani F, Lindozzi M, Miozzi S. Underground neutron flux measurement. Nucl Instrum Methods A. (1988) 272:8714. doi: 10.1016/0168-9002(88)90772-3

7. Satta L, Augusti-Tocco G, Ceccarelli R, Esposito A, Fiore M, Paggi P, et al. Low environmental radiation background impairs biological defence of the yeast Saccharomyces cerevisiae to chemical radiomimetic agents. Mutat Res. (1995) 347:129-33. doi: 10.1016/0165-7992(95)00031-3

8. Satta L, Antonelli F, Belli M, Sapora O, Simone G, Sorrentino E, et al. Influence of a low background radiation environment on biochemical and biological responses in V79 cells. Radiat Environ Biophys. (2002) 41:21724. doi: 10.1007/s00411-002-0159-2

9. Carbone MC, Pinto M, Antonelli F, Amicarelli F, Balata M, Belli M, et al. The Cosmic Silence experiment: on the putative adaptive role of environmental ionizing radiation. Radiat Environ Biophys. (2009) 48:18996. doi: 10.1007/s00411-008-0208-6

10. Hooker AM, Horne R, Morley AA, Sykes PJ. Dose-dependent increase or decrease of somatic intrachromosomal recombination produced by etoposide. Mutation Res. (2002) 500:117-24. doi: 10.1016/s0027-5107(02)00007-6

11. Capece D, Fratini E. The use of pKZ1 mouse chromosomal inversion assay to study biological effects of environmental background radiation. Eur Phys J Plus. (2012) 127:37. doi 10.1140/epjp/i2012-12037-7

\section{AUTHOR CONTRIBUTIONS}

MF, DVer, DVec, and DC performed the LRE experiments. $\mathrm{EF}$ and GE performed the RRE experiments. BD performed the western blot. MB and LI contributed to LRE experiments' setup. PS and LS conceived the study. AT analyzed the data, wrote, and revised the manuscript. FZ, MT, and EA conceived the study, elaborated data, and wrote and revised the manuscript. All authors contributed to the article and approved the submitted version.

\section{FUNDING}

MF and EF were funded by the Centro Studi e Ricerche Enrico Fermi, Rome. This work was supported by the INFN-CSN5 COSMIC SILENCE Experiment.

\section{ACKNOWLEDGMENTS}

The authors would like to thank LNGS and all technical Services for their skilled assistance and support for the experiments.

12. Sykes PJ, Day TK, Swinburne SJ, Lane JM, Morley AA, Hooker AM, et al. In vivo mutagenic effect of very low dose radiation. Dose Response. (2006) 4:309-16. doi: 10.2203/dose-response.06-004.Sykes

13. Day TK, Zeng G, Hooker AM, Bhat M, Scott BR, Turner DR, et al. Extremely low priming doses of $\mathrm{X}$ radiation induce an adaptive response for chromosomal inversions in pKZ1 mouse prostate. Radiat Res. (2006) 166:757-66. doi: 10.1667/RR0689.1

14. Sykes PJ, Morley AA, Hooker AM. The PKZ1 recombination mutation assay: a sensitive assay for low dose studies. Dose Response. (2006) 4:91105. doi: 10.2203/dose-response.05-035.Sykes

15. Zeng G, Day TK, Hooker AM, Blyth BJ, Bhat M, Tilley WD, et al. Nonlinear chromosomal inversion response in prostate after low dose X-radiation exposure. Mutat Res. (2006) 602:65-73. doi: 10.1016/j.mrfmmm.2006.08.002

16. Sykes PJ, Day TK. Requirements for identification of low dose and nonlinear mutagenic responses to ionising radiation. Dose Response. (2007) 5:30814. doi: 10.2203/dose-response.07-018.Sykes

17. Day TK, Zeng G, Hooker AM, Bhat M, Turner DR, Sykes PJ. Extremely low doses of X-radiation can induce adaptive responses in mouse prostate. Dose Response. (2007) 5:315-22. doi: 10.2203/dose-response.07-019.Day

18. Day TK, Zeng G, Hooker AM, Bhat M, Scott BR, Turner DR, et al. Adaptive response for chromosomal inversions in $\mathrm{pKZ1}$ mouse prostate induced by low doses of $\mathrm{X}$ radiation delivered after a high dose. Radiat Res. (2007) 167:682-92. doi: 10.1667/RR0764.1

19. Ormsby RJ, Staudacher AH, Blyth BJ, Bezak E, Sykes PJ. Temporal responses to $\mathrm{X}$-radiation exposure in spleen in the $\mathrm{pKZ1}$ mouse recombination assay. Radiat Res. (2016) 185:623-9. doi: 10.1667/RR14390.1

20. Bannister L, Serran M, Bertrand L, Klokov D, Wyatt H, Blimkie M, et al. Environmentally relevant chronic low-dose tritium and gamma exposures do not increase somatic intrachromosomal recombination in pKZ1 mouse spleen. Radiat Res. (2016) 186:539-48. doi: 10.1667/RR14564.1

21. Kaufmann SH, Desnoyers S, Ottaviano Y, Davidson NE, Poirier GG. Specific proteolytic cleavage of poly(ADP-ribose) polymerase: an early marker of chemotherapy-induced apoptosis. Cancer Res. (1993) 53:3976-85.

22. Tewari M, Quan LT, O’Rourke K, Desnoyers S, Zeng Z, Beidler DR, et al. Yama/CPP32 beta, a mammalian homolog of CED-3, is a CrmAinhibitable protease that cleaves the death substrate poly (ADP-ribose) polymerase. Cell. (1995) 81:801-9. doi: 10.1016/0092-8674(95)90541-3

23. Fischer U, Janicke RU, Schulze-Osthoff K. Many cuts to ruin: a comprehensive update of caspase substrates. Cell Death Differ. (2003) 10:76-100. doi: 10.1038/sj.cdd.4401160 
24. Aubrey BJ, Kelly GL, Janic A, Herold MJ, Strasser A. How does p53 induce apoptosis and how does this relate to p53-mediated tumour suppression? Cell Death Differ. (2018) 25:104-13. doi: 10.1038/cdd.2017.169

25. Fischer M. Census and evaluation of p53 target genes. Oncogene. (2017) 36:3943-56. doi: 10.1038/onc.2016.502

26. White E. Autophagy and p53. Cold Spring Harb Perspect Med. (2016) 6:a026120. doi: 10.1101/cshperspect.a026120

27. Tanida I, Minematsu-Ikeguchi N, Ueno T, Kominami E. Lysosomal turnover, but not a cellular level, of endogenous LC3 is a marker for autophagy. Autophagy. (2005) 1:84-91. doi: 10.4161/auto.1.2.1697

28. Tanida I, Takashi U, Kominami E. LC3 and autophagy. Methods Mol Biol. (2008) 445:77-88. doi: 10.1007/978-1-59745-157-4_4

29. Mullenders L, Atkinson M, Paretzke H, Sabatier L, Bouffler S. Assessing cancer risks of low-dose radiation. Nat Rev Cancer. (2009) 9:596604. doi: 10.1038/nrc2677

30. Tubiana M. Radiation risks in perspective: radiation-induced cancer among cancer risks. Radiat Environ Biophys. (2000) 39:3-16. doi: 10.1007/pl00007682

31. Mothersill C, Seymour C. Radiation-induced non-targeted effects of low doses-what, why and how? Health Phys. (2011) 100:302. doi: 10.1097/hp.0b013e3182080f0c

32. Yonekawa T, Thorburn A. Autophagy and cell death. Essays Biochem. (2013) 55:105-17. doi: 10.1042/bse0550105

33. Bialik S, Dasari SK, Kimchi A. Autophagy-dependent cell deathwhere, how and why a cell eats itself to death. J Cell Sci. (2018) 131:jcs215152. doi: 10.1242/jcs. 215152

34. Denton D, Kumar S. Autophagy-dependent cell death. Cell Death Differ. (2019) 26:605-16. doi: 10.1038/s41418-018-0252-y

35. Verzella D, Pescatore A, Capece D, Vecchiotti D, Ursini MV, Franzoso G, et al. Life, death, and autophagy in cancer: NF-kB turns up everywhere. Cell Death Dis. (2020) 11:210. doi: 10.1038/s41419-020-2399-y
36. Lacroix M, Riscal R, Arena G, Linares LK, Le Cam L. Metabolic functions of the tumor suppressor p53: Implications in normal physiology, metabolic disorders, and cancer. Mol Metab. (2020) 33:2-22. doi: 10.1016/j.molmet.2019.10.002

37. Pietrocola F, Izzo V, Niso-Santano M, Vacchelli E, Galluzzi L, Maiuri $\mathrm{MC}$, et al. Regulation of autophagy by stress-responsive transcription factors. Semin Cancer Biol. (2013) 23:310-22. doi: 10.1016/j.semcancer.2013. 05.008

38. Maiuri MC, Galluzzi L, Morselli E, Keep O, Malik SA, Kroemer G. Autophagy regulation by p53. Curr Opin Cell Biol. (2010) 22:1815. doi: 10.1016/j.ceb.2009.12.001

39. MacLaine NJ, Hupp TR. How phosphorylation controls p53. Cell Cycle. (2011) 10:916-21. doi: 10.4161/cc.10.6.15076

40. Cox ML, Meek DW. Phosphorylation of serine 392 in p53 is a common and integral event during p53 induction by diverse stimuli. Cell Signal. (2010) 22:564-71. doi: 10.1016/j.cellsig.2009. 11.014

Conflict of Interest: The authors declare that the research was conducted in the absence of any commercial or financial relationships that could be construed as a potential conflict of interest.

Copyright (C) 2021 Fischietti, Fratini, Verzella, Vecchiotti, Capece, Di Francesco, Esposito, Balata, Ioannuci, Sykes, Satta, Zazzeroni, Tessitore, Tabocchini and Alesse. This is an open-access article distributed under the terms of the Creative Commons Attribution License (CC BY). The use, distribution or reproduction in other forums is permitted, provided the original author(s) and the copyright owner(s) are credited and that the original publication in this journal is cited, in accordance with accepted academic practice. No use, distribution or reproduction is permitted which does not comply with these terms. 